\title{
Regional anesthesia during COVID-19 pandemic: A mini- review and practical recommendations
}

\author{
Hamidreza Azizi Farsani $^{\circledR}{ }^{\circledR}$, Faranak Behnaz ${ }^{\circledR}$, Zahra Azizi Farsani ${ }^{\circledR}$, Shayesteh Khorasanizadeh ${ }^{1 *}$ \\ ${ }^{1}$ Department of Anesthesiology, Shohada Tajrish Hospital, Shahid Beheshti University of Medical Sciences, Tehran, Iran \\ ${ }^{2}$ Student of Pharmacy School, Shahid Beheshti University of Medical Sciences, Tehran, Iran
}

*Corresponding Author: Shayesteh Khorasanizadeh, Assistant Professor of Department of Anesthesiology, Shohada Tajrish Hospital, Shahid Beheshti University of Medical Sciences, Tehran, Iran. Tel: +982122741174, Email: shayesteh_1343@ yahoo.com

\begin{abstract}
Background and aims: Coronavirus disease 2019 (COVID-19) has posed many challenges for healthcare workers around the world. This study aims to present the most appropriate and evidence-based scientific advice for safe regional anesthesia practice during the COVID-19 pandemic.

Methods: To perform regional anesthesia during respiratory disease outbreaks, several databases were investigated, including PubMed, Google Scholar, Up-to-date, and ScienceDirect. Searches were in English during a timeframe spanning from February 15, 2019, until February 15, 2021, and the applied keywords were "COVID-19 anesthesia", "surgery", and "operating room"

Results: We have focused on definite areas such as workforce and resource planning, modification of clinical setting, preparation of equipment, supplies, and medications, selection of proper personal protective equipment (PPE), cardiorespiratory monitoring of the patient, and sedation and oxygen therapy. Other fields were assessing safe regional anesthesia techniques and monitoring during anesthesia, postanesthesia care, and follow-ups. In these recommendations, keeping the patients safe while protecting healthcare providers from potential exposure to infection is of particular interest.

Conclusion: The safety of healthcare workers and patients during the anesthesia management of suspected and positive COVID-19 cases is of utmost importance. Although there is limited evidence regarding performing regional anesthesia, these presented suggestions summarize the best accessible data and explain some doubts in this respect.

Keywords: COVID-19, Regional anesthesia, Peripheral nerve block, Neuraxial anesthesia, Practical recommendations
\end{abstract}

Received: 2 October 2021, Accepted: 20 November 2021, ePublished: 26 January 2022

\section{Introduction}

Coronavirus disease 2019 (COVID-19) has so far infected 162773940 individuals and killed 3375573 worldwide since 17 May 2021 (1). The most common symptoms include fever, cough, myalgia, fatigue, and dyspnea. Unfortunately, healthcare workers interacting with asymptomatic patients are exposed to a high infection risk daily. Different guidelines and recommendations have so far been published for the anesthesia management of suspected or confirmed COVID-19 patients (2,3). Places, where candidate patients for emergency surgeries are situated before the surgery, are considered to be of high risk, and necessary measures to mitigate the infection level in these places are particularly important (4). One strategy to reduce the exposure level is to avoid aerosol-producing techniques such as airway management techniques usually performed during surgery. General anesthesia leads to aerosol production through intervention in the airway, which exposes the healthcare team to the COVID-19 transmission risk during intubation and extubation $(5,6)$. It is believed that the possibility of acute respiratory infection transmission is higher for patients requiring intubation compared with patients who are not subjected to intubation (7). Intubation for a COVID-19 positive patient should ideally be conducted in a negative pressure room, which may be unavailable in all places or situations (8).

Anesthesiologists recommended using local or regional anesthesia practical and safe during the critical care of patients with coronavirus $(9,10)$. Compared to general anesthesia, the advantages of regional anesthesia include a reduction in aerosol-generating procedures, an increase in safety and the saving of the time, resource, and financial costs of personal protective equipment (PPE), preservation of immune function, minimization of direct contact with caregivers, and earlier discharge (11).

Regional anesthesia is the technique of choice since it eliminates aerosol-producing procedures, decreases costs, and provides safe anesthesia. Despite previous epidemics such as severe acute respiratory syndrome (SARS) in 2003 and Middle East respiratory syndrome (MERS) in 2012, a few evidence-based guidelines are available for regional anesthesia, and an immediate need for such guidelines has been expressed by anesthesiologists (12-14). A statement

(C) 2022 The Author(s); Published by Shahrekord University of Medical Sciences. This is an open-access article distributed under the terms of the Creative Commons Attribution License (http://creativecommons.org/licenses/by/4.0), which permits unrestricted use, distribution, and reproduction in any medium, provided the original work is properly cited. 
has recently been published by the American Society of Regional Anesthesia and Pain Medicine and the European Society of Regional Anesthesia and Pain Therapy Joint Committee for regional anesthesia during the COVID-19 pandemic (14). This study seeks to present the most appropriate and evidence-based scientific suggestions for safe regional anesthesia practice during the COVID-19 outbreak.

\section{Methods}

In this study, the evaluated areas of interest included workforce and resource planning, clinical setting modification, preparation of equipment, supplies, and medications, selection of proper PPE, and the cardiorespiratory monitoring of the patient. Other areas were sedation and oxygen therapy, assessment of safe regional anesthesia techniques, and monitoring during anesthesia, post-anesthesia care, and follow-up.

To perform regional anesthesia during respiratory disease outbreaks several sources were examined, including PubMed, Google Scholar, Up-to-date, and ScienceDirect. In addition, searches were in English and performed from February 15, 2019 to February 15, 2021, and the used keywords were "COVID-19 anesthesia", "surgery", and "operating room". This work sought to study all available information regarding regional anesthesia during the COVID-19 or similar epidemics. The full texts of all the selected papers were carefully reviewed, and contents related to regional anesthesia were extracted accordingly. Considering the collected evidence, the following recommendations were obtained by common agreement after discussion with specialists.

\section{Recommendation}

\section{Planning and preparation}

Key considerations during pre-operative regional anesthesia were as follows (Figure 1):

All elective surgeries must be deferred to reduce the risk of infection to patients and healthcare providers, save the capacity of the healthcare system, and increase for the opportunity of improved outcomes $(15,16)$.

In cases of suspected COVID-19, regional anesthesia is the first choice if possible. Preoperative evaluations should include COVID-19 screening or testing for

\begin{tabular}{|l|l|}
\hline - Planning and preparation \\
\hline - Operating room \\
\hline - Monitoring \\
\hline - Personal protective equipment \\
\hline - Sedation and oxygen therapy \\
\hline - Equipment \\
\hline - Monitoring and anesthesia management \\
\hline 8 & - Postoperative care \\
\hline - Follow-up \\
\hline
\end{tabular}

Figure 1. Key Considerations during pre-operative regional anesthesia. patients who are not known to have COVID-19, and COVID-19-related risk assessments should be performed for all patients. The risks of perioperative morbidity and mortality may be increased in patients with COVID-19. It is noteworthy that preoperative evaluations should be performed according to regional anesthesia guidelines. It is best to conduct the assessment immediately before the surgery by electronic files and video calls $(17,18)$.

All suspected or symptomatic cases with a negative polymerase chain reaction (PCR) test should be considered as positive and the safety guidelines must be followed completely. A small case study has recommended that spinal anesthesia can be performed safely in COVID-19 patients without any significant hemodynamic or infective side effects. Many COVID-19 patients are anticoagulated, affecting the timing and decision to use neuro axial anesthesia or deep peripheral nerve blocks (PNB). The most experienced anesthesiologist should perform regional anesthesia considering the contraindication and appropriate use of safe tactics (19-21).

Other important measures are performing a neurological examination, obtaining informed consent, considering the patient's thrombocytopenia due to its high prevalence, and bringing the patient with a surgical mask into the operating room (22). A platelet number equal to or above 75000 has been proposed as an adequate level for the neuraxial technique in pregnant women, and the platelet performance is normal in this case.

\section{Operating room}

The surgery of patients with positive COVID-19 test results or suspicious must be performed in a small operating room with negative pressure whenever possible $(18,23,24)$. In case of unavailability, the bioengineering department must be asked to increase the air conditioning of the room. For instance, a portable high-efficiency particulate absorbing filter system should be used in this condition $(19,25,26)$. Further, a standard operating room with a minimum of 15 air exchanges/hour should be prepared because $99 \%$ of infective aerosols are eliminated within 20 minutes by this method $(23,24)$.

\section{Monitoring}

Some common recommendations include the monitoring of the heart rate, peripheral saturation of oxygen (Spo2), noninvasive blood pressure, conscious levels, symptoms of local anesthetic toxicity, respiratory conditions, and depth and block levels in case of COVID-19 during the regional block $(27,28)$.

\section{Personal protective equipment}

In regional anesthesia, aerosol production is less compared to general anesthesia. However, due to secretions, aerosols, or micro-droplets produced by coughing, preventive measures are necessary, including the use of minimum personnel, surgical mask, goggles or shield, cap and waterresistant gown, two pairs of gloves, and plastic foot cover. 
To prevent COVID-19 transmission, patients must wear surgical masks, although wearing N95 or FFP2/3 masks is generally not necessary. However, it may be prudent for prolonged contact with a COVID-19 patient in a secure location. The health care provider should consider wearing and taking off PPE to prevent contamination $(22,23)$.

\section{Sedation and oxygen therapy}

The administration of sedative drugs must be avoided whenever possible due to respiratory reductions and the need for airway intervention, and careful respiratory monitoring must be performed in case of their use. In oxygen therapy, the aim is to maintain the Spo2 in a normal range with the minimum oxygen flow since a high flow leads to aerosol production and viral transmission. Oxygen cannula must be inserted under the face mask and an oxygen mask should be used over the surgical mask of the patient $(21,22)$.

\section{Equipment}

The unnecessary equipment in the room must be minimized and single-use items should be provided in this situation. In addition, equipment and required medication should be prepared and packaged for immediate postoperative care in plastic bags, and various equipment such as ultrasound, anesthesia, and suction machines should be protected with a plastic cover during various practices, and then they should be disinfected using antiseptic solutions after transferring the patient to the surgical ward.

\section{Monitoring and anesthesia management}

Performing safe regional anesthesia prevents various risks such as emergency conversion to general anesthesia, evaluation of local anesthetic systemic toxicity, and use of epidural blood patch administration for prolonged postdural puncture headache. Moreover, using sonography and safe local anesthetic by an experienced anesthesiologist prevents these complications. Additionally, selecting a proper location for PNB plays a role in lowering these complications. For example, using axillary block instead of inter scalene block prevents phrenic nerve paralysis and pneumothorax. Before performing surgery, the success of neuro axial anesthesia and PNB must be completely tested to minimize the risk of conversion to general anesthesia $(22,26,27)$.

\section{Postoperative care}

The monitoring and recovery of the patient must be performed in the operating room to prevent the spread of infection to other places. Then, the patient must be transferred to the related unit. Considering that the risk of viral transmission is high when removing protective equipment, additional time must be considered for wearing and taking out the protective equipment.

After the surgery, special attention should be paid to controlling post-operative pain. In addition, it is mandatory to know the current recommendations for the safe use of non-opioid analgesics, opioid analgesics, and interventional pain management techniques, especially during the pandemic $(27,29)$.

\section{Follow-up}

To prevent the spread of infection, the patient's condition should be remotely followed up via telemedicine because the patients and physicians should not meet each other, thus telemedicine and/or video calls are becoming increasingly common and considered acceptable media for patient healthcare workers (30).

\section{Implications}

Overall, 337 papers were found in the reference search, and after screening their titles and abstracts, 31 papers were selected for the complete review of their texts. Recommendations and guidelines for handling suspected or confirmed cases of COVID-19 were briefly presented (Figure 2) based on these papers and extraction of their results (31).

\section{Conclusion}

The safety of healthcare workers and patients during the anesthesia management of suspected and positive COVID-19 cases is of utmost importance. Although evidence for performing regional anesthesia is limited, the presented suggestions summarize the best accessible data and explain some doubts. The best strategy is to postpone elective surgeries in suspected or confirmed individuals until full recovery. The presented recommendations apply to an individual who tested positive or suspected of the COVID-19 infection.

\section{Study limitations and future instructions}

It is believed that the limitations and criticisms of regional anesthesia related to COVID-19 should be further considered, and the advantages and criticisms of regional anesthesia in the management of surgical patients in the pandemic age should be evaluated accordingly (32).

Although the COVID-19 pandemic is not the first respiratory tract infectious diesis over the past two decades, the lack of strong evidence hinders providing any robust scientific recommendation. Individual experiences are significant during a disease outbreak for establishing healthcare programs. A successful experience in the SARS epidemic indicates that the participation of healthcare personnel at the frontline of the fight against the diseases and the use of a theory-based approach are helpful for the formulation of a risk controlling structure and management instructions for the safe performance of aerosol-producing methods. A similar effort is needed for providing evidence-based scientific suggestions about regional anesthesia. Future knowledge about the trend of this disease may change our recommendations.

Authors' Contributions

HAF designed the study, analyzed the data, and revised the 

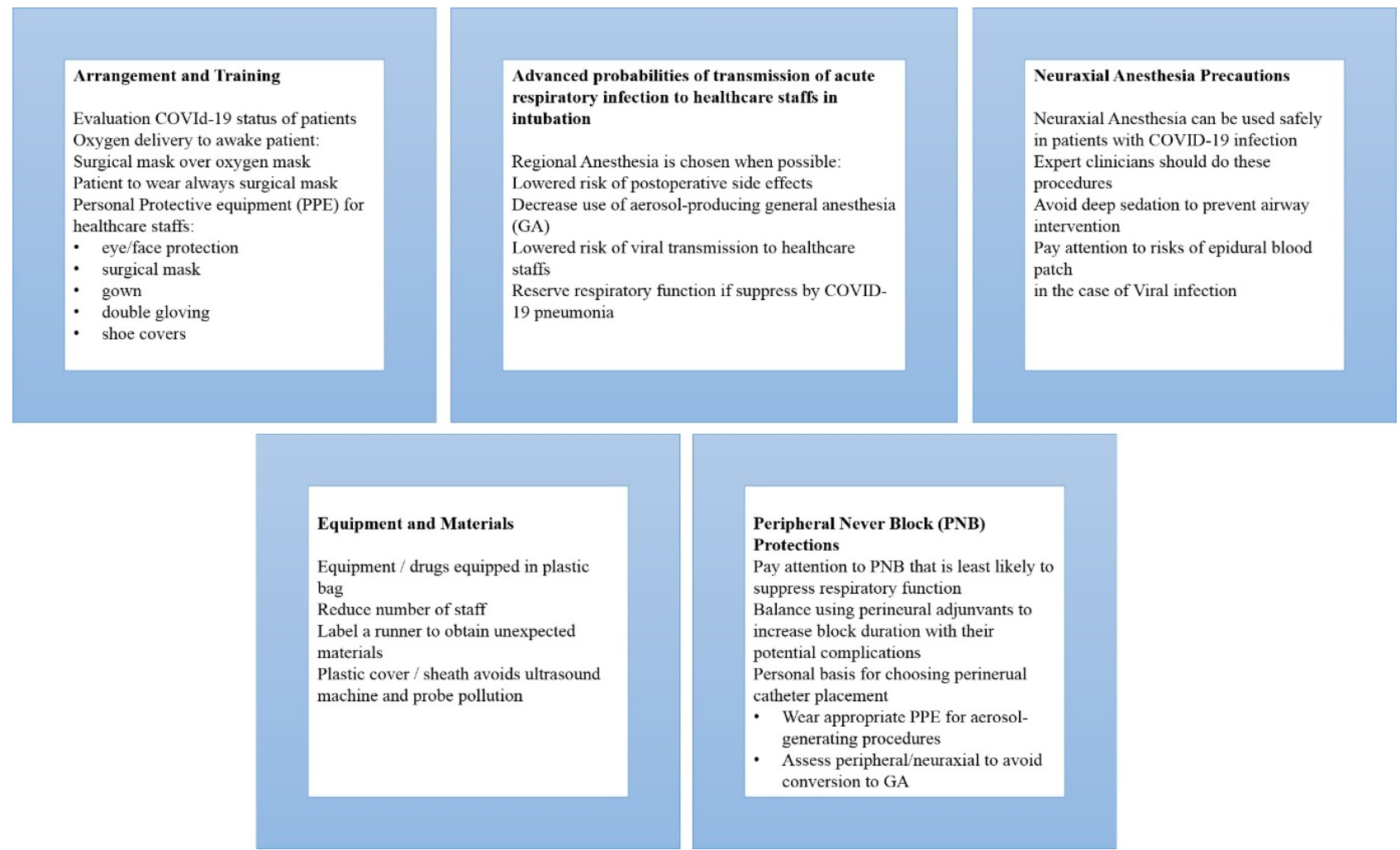

Figure 2. COVID-19: suggestions for regional anesthesia: summary of present recommendations for the performance of regional anesthesia for COVID-19 positive patients or persons under study. Note. COVID-19: Coronavirus disease 19.

paper. SK contributed to the search of databases, title and abstract screening, and data extraction. ZAF contributed to the search of databases and revised the paper. FB revised the final manuscript.

\section{Conflict of Interests}

There is no conflict of interests.

Ethical Approval

Not applicable.

Funding/Support

None declared.

\section{References}

1. World Health Organization (WHO). WHO Coronavirus (COVID-19) Dashboard. WHO; 2021

2. Sanche $\mathrm{S}$, Lin $\mathrm{YT}, \mathrm{Xu} \mathrm{C}$, Romero-Severson $\mathrm{E}$, Hengartner $\mathrm{N}$, Ke R. High contagiousness and rapid spread of severe acute respiratory syndrome coronavirus 2. Emerg Infect Dis. 2020;26(7):1470-7. doi: 10.3201/eid2607.200282.

3. Tran K, Cimon K, Severn M, Pessoa-Silva CL, Conly J. Aerosol generating procedures and risk of transmission of acute respiratory infections to healthcare workers: a systematic review. PLoS One. 2012;7(4):e35797. doi: 10.1371/journal. pone.0035797.

4. Stahel PF. How to risk-stratify elective surgery during the COVID-19 pandemic? Patient Saf Surg. 2020;14:8. doi: 10.1186/s13037-020-00235-9.

5. Schwartz J, King CC, Yen MY. Protecting health care workers during the COVID-19 coronavirus outbreak: lessons from Taiwan's SARS response. Clin Infect Dis. 2020;71:858-60.

6. Imperial College London COVID-19 Response Team. Report 3: Transmissibility of 2019-nCoV. 2020. https:// www.imperial.ac.uk/media/imperial-college/medicine/ sph/ide/gida-fellowships/Imperial-College-COVID19transmissibility-25-01-2020.

7. Wax RS, Christian MD. Practical recommendations for critical care and anesthesiology teams caring for novel coronavirus (2019-nCoV) patients. Can J Anaesth. 2020; 67: 568-76

8. Uppal V, Sondekoppam RV, Landau R, El-Boghdadly K, Narouze S, Kalagara HKP. Neuraxial anaesthesia and peripheral nerve blocks during the COVID-19 pandemic: a literature review and practice recommendations. Anaesthesia. 2020;75(10):1350-63. doi: 10.1111/anae.15105.

9. Warren J, Sundaram K, Anis H, Kamath AF, Mont MA, Higuera CA, et al. Spinal anesthesia is associated with decreased complications after total knee and hip arthroplasty. J Am Acad Orthop Surg. 2020;28(5):e213-e21. doi: 10.5435/ jaaos-d-19-00156.

10. von Ungern-Sternberg BS, Boda K, Chambers NA, Rebmann C, Johnson C, Sly PD, et al. Risk assessment for respiratory complications in paediatric anaesthesia: a prospective cohort study. Lancet. 2010;376(9743):773-83. doi: 10.1016/s01406736(10)61193-2.

11. Altiparmak B, Korkmaz Toker M, Uysal Al, Gümüş Demi Rbi Lek S. Regional anesthesia in patients with suspected COVID-19 infection. Reg Anesth Pain Med. 2021;46(1):91-2. doi: 10.1136/rapm-2020-101477.

12. Zhong Q, Liu YY, Luo Q, Zou YF, Jiang HX, Li H, et al. Spinal anaesthesia for patients with coronavirus disease 2019 and possible transmission rates in anaesthetists: retrospective, single-centre, observational cohort study. $\mathrm{Br} J$ Anaesth. 2020;124(6):670-5. doi: 10.1016/j.bja.2020.03.007.

13. Bauer ME, Bernstein K, Dinges E, Delgado C, El-Sharawi N, Sultan $\mathrm{P}$, et al. Obstetric anesthesia during the COVID-19 pandemic. Anesth Analg. 2020;131(1):7-15. doi: 10.1213/ ane.0000000000004856.

14. Bauer ME, Chiware R, Pancaro C. Neuraxial procedures in COVID-19-positive parturients: a review of current reports. Anesth Analg. 2020;131(1):e22-e4. doi: 10.1213/ ane.0000000000004831.

15. Banik RK, Ulrich A. Evidence of short-range aerosol transmission of SARS-CoV-2 and call for universal airborne precautions for anesthesiologists during the COVID-19 
pandemic. Anesth Analg. 2020;131(2):e102-e4. doi: 10.1213/ ane.0000000000004933.

16. Zhao S, Ling $\mathrm{K}$, Yan $\mathrm{H}$, Zhong L, Peng $\mathrm{X}$, Yao $\mathrm{S}$, et al. Anesthetic management of patients with COVID 19 infections during emergency procedures. J Cardiothorac Vasc Anesth. 2020;34(5):1125-31. doi: 10.1053/j.jvca.2020.02.039.

17. Lie SA, Wong SW, Wong LT, Wong TGL, Chong SY. Practical considerations for performing regional anesthesia: lessons learned from the COVID-19 pandemic. Can J Anaesth. 2020;67(7):885-92. doi: 10.1007/s12630-020-01637-0.

18. Yek JLJ, Kiew SCA, Ngu JC, Lim JGC. Perioperative considerations for COVID-19 patients: lessons learned from the pandemic -a case series. Korean J Anesthesiol. 2020;73(6):557-61. doi: 10.4097/kja.20182.

19. Tang LY, Wang J. Anesthesia and COVID-19: what we should know and what we should do. Semin Cardiothorac Vasc Anesth. 2020;24(2):127-37. doi: 10.1177/1089253220921590.

20. De Simone B, Chouillard E, Sartelli M, Biffl WL, Di Saverio $\mathrm{S}$, Moore EE, et al. The management of surgical patients in the emergency setting during COVID-19 pandemic: the WSES position paper. World J Emerg Surg. 2021;16(1):14. doi: 10.1186/s13017-021-00349-0.

21. Wu Z, McGoogan JM. Characteristics of and important lessons from the coronavirus disease 2019 (COVID-19) outbreak in China: summary of a report of 72314 cases from the Chinese Center for Disease Control and Prevention. JAMA. 2020;323(13):1239-42. doi: 10.1001/jama.2020.2648.

22. Aminnejad R, Shafiee $H$. Is regional anesthesia safe enough in suspected or confirmed COVID-19 patients? ACS Chem Neurosci. 2020;11(9):1371. doi: 10.1021/ acschemneuro.0c00146.

23. Macfarlane AJR, Harrop-Griffiths W, Pawa A. Regional anaesthesia and COVID-19: first choice at last? Br J Anaesth. 2020;125(3):243-7. doi: 10.1016/j.bja.2020.05.016.

24. Peng PWH, Ho PL, Hota SS. Outbreak of a new coronavirus: what anaesthetists should know. Br J Anaesth. 2020;124(5):497501. doi: 10.1016/j.bja.2020.02.008.

25. Al-Benna S. Negative pressure rooms and COVID-19. J Perioper Pract. 2021;31(1-2):18-23. doi: 10.1177/1750458920949453.

26. Bampoe S, Odor PM, Lucas DN. Novel coronavirus SARSCoV-2 and COVID-19. Practice recommendations for obstetric anaesthesia: what we have learned thus far. Int J Obstet Anesth. 2020;43:1-8. doi: 10.1016/j.ijoa.2020.04.006.

27. Uppal V, Sondekoppam RV, Landau R, El-Boghdadly K, Narouze S, Kalagara HKP. Neuraxial anaesthesia and peripheral nerve blocks during the COVID-19 pandemic: a literature review and practice recommendations. Anaesthesia. 2020;75(10):1350-63. doi: 10.1111/anae.15105.

28. Lie SA, Wong SW, Wong LT, Wong TGL, Chong SY. Practical considerations for performing regional anesthesia: lessons learned from the COVID-19 pandemic. Can J Anaesth. 2020;67(7):885-92. doi: 10.1007/s12630-020-01637-0.

29. Cohen SP, Baber ZB, Buvanendran A, McLean BC, Chen $Y$, Hooten WM, et al. Pain management best practices from multispecialty organizations during the COVID-19 pandemic and public health crises. Pain Med. 2020;21(7):1331-46. doi: 10.1093/pm/pnaa127.

30. Williams AM, Bhatti UF, Alam HB, Nikolian VC. The role of telemedicine in postoperative care. Mhealth. 2018;4:11. doi: 10.21037/mhealth.2018.04.03.

31. Herman JA, Urits I, Kaye AD, Urman RD, Viswanath O. COVID-19: Recommendations for regional anesthesia. J Clin Anesth. 2020;65:109885. doi: 10.1016/j. jclinane.2020.109885.

32. Cappelleri G, Fanelli A, Ghisi D, Russo G, Giorgi A, Torrano $\mathrm{V}$, et al. The role of regional anesthesia during the SARSCoV2 pandemic: appraisal of clinical, pharmacological and organizational aspects. Front Pharmacol. 2021;12:574091. doi: 10.3389/fphar.2021.574091. 